\title{
Measuring and Computing Database Inconsistency via Repairs
}

\author{
Leopoldo Bertossi ${ }^{\star}$ \\ Carleton University, Ottawa, Canada \& Relational ${ }^{\mathrm{AI}}$, Inc.
}

\begin{abstract}
We propose a generic numerical measure of inconsistency of a database with respect to a set of integrity constraints. It is based on an abstract repair semantics. A particular inconsistency measure associated to cardinality-repairs is investigated; and we show that it can be computed via answer-set programs.
\end{abstract}

Keywords: Integrity constraints in databases, inconsistent databases, database repairs, inconsistency measures

Intuitively, a relational database may be more or less consistent than others databases for the same schema with the same integrity constraints (ICs). This comparison can be accomplished by assigning a measure of inconsistency to a database. The inconsistency degree of a database $D$ with respect to (wrt.) a set of ICs $\Sigma$ should depend on how complex it is to restore consistency; or more technically, on the class of repairs of $D$ wrt. $\Sigma$. For this we can apply concepts and results on database repairs (cf. [2] for a survey and references). Our stand on degrees of inconsistency is that they depend on how consistency is restored, i.e. involving the admissible repair actions and how close we want stay to the instance at hand. This short communication shows preliminary research on possible ways to make these ideas concrete, by defining and analyzing a measure of inconsistency of a relational database instance, and providing mechanisms for computing this measure using answer-set programming (ASP) [5].

Database Repairs. When a database instance $D$ does not satisfy its intended ICs, it is repaired, by deleting or inserting tuples from/into the database. An instance obtained in this way is a repair of $D$ if it satisfies the ICs and departs in a minimal way from $D$ [2]. In this work, just to fix ideas, we consider mostly ICs that can only be solved by tuple deletions, e.g. most prominently, denial constraints (DCs) and functional dependencies (FDs). DCs are logical formulas of the form $\neg \exists \bar{x}\left(P_{1}\left(\bar{x}_{1}\right) \wedge \cdots \wedge P_{m}\left(\bar{x}_{m}\right)\right)$, where $\bar{x}=\bigcup \bar{x}_{i}$; and FDs are of the form $\neg \exists \bar{x}\left(P\left(\bar{v}, \bar{y}_{1}, z_{1}\right) \wedge P\left(\bar{v}, \bar{y}_{2}, z_{2}\right) \wedge z_{1} \neq z_{2}\right)$, with $\bar{x}=\bar{y}_{1} \cup \bar{y}_{2} \cup \bar{v} \cup\left\{z_{1}, z_{2}\right\}$. We treat FDs as DCs. A database is inconsistent wrt. a set of ICs $\Sigma$ when $D$ does not satisfy $\Sigma$, denoted $D \not \models \Sigma$.

Example 1. The DB $D=\{P(a), P(e), Q(a, b), R(a, c)\}$ is inconsistent with respect to the (set of) denial constraints (DCs) $\kappa_{1}: \neg \exists x \exists y(P(x) \wedge Q(x, y))$, and $\kappa_{2}$ : $\neg \exists x \exists y(P(x) \wedge R(x, y))$. Here, $D \forall \neq\left\{\kappa_{1}, \kappa_{2}\right\}$.

A subset-repair, in short an $S$-repair, of $D$ wrt. the set of DCs is a $\subseteq$-maximal subset of $D$ that is consistent, i.e. no proper superset is consistent. The following are S-repairs: $D_{1}=\{P(e), Q(a, b), R(a, c)\}$ and $D_{2}=\{P(e), P(a)\}$. Under this repair semantics, both repairs are equally acceptable. A cardinality-repair, in short a $C$-repair, is a maximum-cardinality S-repair. $D_{1}$ is the only C-repair.

For an instance $D$ and a set $\Sigma$ of DCs, the sets of S-repairs and C-repairs are denoted with $\operatorname{Srep}(D, \Sigma)$ and $\operatorname{Crep}(D, \Sigma)$, resp. It holds: $\operatorname{Crep}(D, \Sigma) \subseteq \operatorname{Srep}(D, \Sigma)$. More generally, for a set $\Sigma$ of ICs, not necessarily DCs, they can be defined by (cf. [2]):

\footnotetext{
* Member of the "Millenium Institute for Foundational Research on Data" (IMFD, Chile). Email: bertossi@scs.carleton.ca. Research supported by NSERC Discovery Grant \#06148.
} 
$\operatorname{Srep}(D, \Sigma)=\left\{D^{\prime} \quad: \quad D^{\prime} \models \Sigma\right.$, and $D \triangle D^{\prime}$ is minimal under set inclusion $\}$, and $\operatorname{Crep}(D, \Sigma)=\left\{D^{\prime}: D^{\prime} \models \Sigma\right.$, and $D \triangle D^{\prime}$ is minimal in cardinality $\}$. Here, $D \triangle D^{\prime}$ is the symmetric set difference $\left(D \backslash D^{\prime}\right) \cup\left(D^{\prime} \backslash D\right)$.

Repair Semantics and Inconsistency Degrees. In general terms, a repair semantics $S$ for a schema $\mathcal{R}$ that includes a set $\Sigma$ of ICs assigns to each instance $D$ for $\mathcal{R}$ (which may not satisfy $\Sigma$ ), a class $\operatorname{Rep}^{\mathrm{S}}(D, \Sigma)$ of S-repairs of $D$ wrt. $\Sigma$, which are instances of $\mathcal{R}$ that satisfy $\Sigma$ and depart from $D$ according to some minimization criterion. Several repair semantics have been considered in the literature, among them and beside those above, prioritized repairs [15], and attribute-based repairs that change attribute values by other data values, or by a null value, NULL, as in SQL databases (cf. [1, 2]).

According to our take on how a database inconsistency degree depends on database repairs, we define the inconsistency degree of an instance $D$ wrt. a set of ICs $\Sigma$ in relation to a given repair semantics $\mathrm{S}$, as the distance from $D$ to the class $\operatorname{Rep}^{\mathrm{S}}(D, \Sigma)$ :

$$
\text { inc-deg } \mathrm{S}(D, \Sigma):=\operatorname{dist}\left(D, \operatorname{Rep}^{\mathrm{S}}(D, \Sigma)\right) \text {. }
$$

This is an abstract measure that depends on $\mathrm{S}$ and a chosen distance function dist, from a world to a set of possible worlds. Under the assumption that any repair semantics should return $D$ when $D$ is consistent wrt. $\Sigma$ and $\operatorname{dist}(D,\{D\})=0$, a consistent instance $D$ should have 0 as inconsistency degree 1

Notice that the class $\operatorname{Rep}^{\mathrm{S}}(D, \Sigma)$ might contain instances that are not sub-instances of $D$, for example, for different forms of inclusion dependencies (INDs) we may want to insert tuples 2 or even under DCs, we may want to appeal to attribute-based repairs. In the following we consider only repairs that are sub-instances of the given instance. Still this leaves much room open for different kinds of repairs. For example, we may prefer to delete some tuples over others [15]. Or, as in database causality [13, 3], the database can be partitioned into endogenous and exogenous tuples, assuming we have more control on the former, or we trust more the latter; and we prefer endogenous repairs that delete preferably (only or preferably) endogenous tuples [1].

An Inconsistency Measure. Here we consider a concrete instantiation of (1), and to fix ideas, only DCs. For them, the repair semantics $\operatorname{Srep}(D, \Sigma)$ and $\operatorname{Crep}(D, \Sigma)$ are particular cases of repair semantics $\mathrm{S}$ where each $D^{\prime} \in \operatorname{Rep}^{\mathrm{S}}(D, \Sigma)$ is maximally contained in $D$. On this basis, we can define:

$$
\begin{aligned}
\text { inc-deg }^{\mathrm{S}, g_{3}}(D, \Sigma) & :=\operatorname{dist}^{g_{3}}\left(D, \operatorname{Rep}^{\mathrm{S}}(D, \Sigma)\right):=\frac{|D|-\max \left\{\left|D^{\prime}\right|: D^{\prime} \in \operatorname{Rep}^{\mathrm{S}}(D, \Sigma)\right\}}{|D|} \\
& =\frac{\min \left\{\left|D \backslash D^{\prime}\right|: D^{\prime} \in \operatorname{Rep}^{\mathrm{S}}(D, \Sigma)\right\}}{|D|}
\end{aligned}
$$

inspired by distance $g_{3}$ in [11] to measure the degree of violation of an FD by a database, whose satisfaction is restored through tuple deletions 3 This measure can be applied more generally as a "quality measure", not only in relation to inconsistency, but also whenever possibly several intended "quality versions" of a dirty database exist, e.g. as determined by additional contextual information [4].

\footnotetext{
${ }^{1}$ Abstract distances between two point-sets are investigated in [8], with their computational properties. Our setting is a particular case.

${ }^{2}$ For INDs repairs based only on tuple deletions can be considered [6].

${ }^{3}$ Other possible measures for single FDs and relationships between them can be found in [11].
} 
Example 2. (ex. 1 1 cont.) Here, $\operatorname{Srep}(D, \Sigma)=\left\{D_{1}, D_{2}\right\}$, and $\operatorname{Crep}(D, \Sigma)=\left\{D_{1}\right\}$. They provide the inconsistency degrees:

$$
\begin{aligned}
& \text { inc-deg }{ }^{s, g_{3}}(D, \Sigma):=\frac{4-\max \left\{\left|D^{\prime}\right|: D^{\prime} \in \operatorname{Srep}(D, \Sigma)\right\}}{4}=\frac{4-\left|D_{1}\right|}{4}=\frac{1}{4}, \\
& \text { inc- } \operatorname{deg}^{c, g_{3}}(D, \Sigma):=\frac{4-\max \left\{\left|D^{\prime}\right|: D^{\prime} \in \operatorname{Crep}(D, \Sigma)\right\}}{4}=\frac{4-\left|D_{1}\right|}{4}=\frac{1}{4}
\end{aligned}
$$

respectively.

It holds $\operatorname{Crep}(D, \Sigma) \subseteq \operatorname{Srep}(D, \Sigma)$, but $\max \left\{\left|D^{\prime}\right|: D^{\prime} \in \operatorname{Crep}(D, \Sigma)\right\}=$ $\max \left\{\left|D^{\prime}\right|: D^{\prime} \in \operatorname{Srep}(D, \Sigma)\right\}$, so it holds inc-deg ${ }^{s, g_{3}}(D, \Sigma)=\operatorname{inc}^{-\operatorname{deg}^{c,} g_{3}}(D, \Sigma)$. These measures always takes a value between 0 and 1 . The former when $D$ is consistent (so it itself is its only repair). The measure takes the value 1 only when $\operatorname{Rep}^{\mathrm{S}}(D, \Sigma)=\emptyset$ (assuming that $\max \left\{\left|D^{\prime}\right|: D^{\prime} \in \emptyset\right\}=0$ ), i.e. the database is irreparable, which is never the case for DCs and S-repairs: there is always an S-repair. However, it could be irreparable with different, but related repair semantics. For example, when we accept only endogenous repairs and none of them exists [3].

Example 3. (ex. 2 cont.) Assume $D$ is partitioned into endogenous and exogenous tuples, say resp. $D=D^{n} \dot{\cup} D^{x}$, with $D^{n}=\{Q(a, b), R(a, c)\}$ and $D^{x}=\{P(a), P(e)\}$. In this case, the endogenous-repair semantics that allows only a minimum number of deletions of endogenous tuples, defines the class of repairs: $\operatorname{Srep}^{c, n}(D, \Sigma)=\left\{D_{2}\right\}$, with $D_{2}$ as above. In this case 4 inc- $\operatorname{deg}^{c, n, g_{3}}(D, \Sigma)=\frac{4-2}{4}=\frac{1}{2}$. Similarly, if now $D^{n}=\{P(a), Q(a, b)\}$ and $D^{x}=\{P(e), R(a, c)\}$, there are no endogenous repairs, and inc-deg $g^{c, n, g_{3}}(D, \Sigma)=1$.

ASP-Based Computation of the Inconsistency Measure. We concentrate on measure inc-deg ${ }^{c, g_{3}}(D, \Sigma)$ (cf. (4)). More generally, we can start from inc-deg ${ }^{s, g_{3}}(D, \Sigma)$, which can be computed through the maximum cardinality of an S-repair for $D$ wrt. $\Sigma$, or, equivalently, using the cardinality of a (actually, every) repair in $\operatorname{Crep}(D, \Sigma)$. In its turn, this can be done 5 through compact specifications of repairs by means of ASPs. We just show an example.

Example 4. (ex. 1 cont.) For technical convenience, we insert global tuple-ids in $D$, i.e. $D=\{P(1, e), Q(2, a, b), R(3, a, c), P(4, a)\}$. It is possible to write an answerset program, a repair program, $\Pi$ whose stable models $\mathcal{M}_{1}, \mathcal{M}_{2}$ are correspondence with the repairs $D_{1}, D_{2}$, resp., namely $\mathcal{M}_{1}=\left\{P^{\prime}(1, e, \mathrm{~s}), Q^{\prime}(2, a, b, \mathrm{~s}), R^{\prime}(3, a, c, \mathrm{~s})\right.$, $\left.P^{\prime}(4, a, \mathrm{~d})\right\} \cup D$ and $\mathcal{M}_{2}=\left\{P^{\prime}(1, e, \mathrm{~s}), P^{\prime}(4, a, \mathrm{~s}), Q^{\prime}(2, a, b, \mathrm{~d}), R^{\prime}(3, a, c, \mathrm{~d})\right\} \cup D$, where the primed predicates are nicknames for the original ones, and the annotations constants $\mathrm{s}, \mathrm{d}$ indicate that the tuple stays or is deleted in/from the database, resp. [1, 7]

Now, to compute inc-deg $g^{c, g_{3}}(D, \Sigma)$, for the C-repair semantics, we can add rules to $\Pi$ to collect the tids of tuples deleted from the database: $\operatorname{Del}(t) \leftarrow R^{\prime}(t, x, y, \mathrm{~d})$, similarly for $Q^{\prime}$ and $P^{\prime}$. And next, a rule to count the deleted tuples, say: $\operatorname{NumDel}(n) \leftarrow$ $\#$ count $\{t: \operatorname{Del}(t)\}=n$. For example, program $\Pi$ with the new rules added will see

\footnotetext{
${ }^{4}$ For certain forms of prioritized repairs, such as endogenous repairs, the normalization coefficient $|D|$ might be unnecessarily large. In this particular case, it might be better to use $\left|D^{n}\right|$.

${ }^{5}$ This approach was followed in [1] to compute maximum responsibility degrees of database tuples as causes for violations of DCs, appealing to a causality-repair connection [3].
} 
the original stable model $\mathcal{M}_{1}$ extended with the atoms $\operatorname{Del}(4), N u m \operatorname{Del}(1)$. Similarly for $\mathcal{M}_{2}$.

Since the stable models of the program capture the S-repairs, i.e. $\subseteq$-maximal and consistent sub-instances of $D$, we can add to $\Pi$ weak program constraints [12], such as ": $\sim P(t, x), P^{\prime}(t, x, \mathrm{~d})$ " ( similarly for $R$ and $Q$ ). They have the effect of eliminating the models of the original program that do not violate them in a minimum way. More precisely, they make us keep only the stable models of the original program that minimize the number of satisfactions of the constraint bodies. In our case, only the models (repairs) that minimize the number of tuple deletions are kept, i.e. models that correspond to C-repairs of $D$. In this example, only (the extended) $\mathcal{M}_{1}$ remains. The value for NumDel in any of them can be used to compute inc-deg ${ }^{c, g_{3}}(D, \Sigma)$. There is no need to explicitly compute all stable models, their sizes, and compare them. This value can be obtained by means of the query, "NumDel(x)?", answered by the program under the brave semantics (returning an answer from some stable model).

Discussion. There are many open issues, among them exploring other inconsistency measures, e.g. based on the Jaccard distance [14]. Several measures have been considered in knowledge representation [10, 16, 9], mostly for the propositional case. It would be interesting to analyze the general properties of those measures that are closer to database applications, along the lines of [8]; and their relationships. For each measure it becomes relevant to investigate the complexity of its computation, in particular, in data complexity (databases may have exponentially many repairs, in data [2])6 Actually, it is possible to prove that computing inc- $\operatorname{deg}^{c, g_{3}}(D, \Sigma)$ is complete for the functional class $F P^{N P(\log (n))}$ in data, and this both for sets $\Sigma$ of DCs and of FDs.

\section{References}

[1] Bertossi, L. Characterizing and Computing Causes for Query Answers in Databases from Database Repairs and Repair Programs. Corr Arxiv cs.DB/1712.01001. Proc. FoIKs, 2018.

[2] Bertossi, L. Database Repairing and Consistent Query Answering. Morgan \& Claypool, Synthesis Lectures on Data Management, 2011.

[3] Bertossi, L. and Salimi, B. From Causes for Database Queries to Repairs and Model-Based Diagnosis and Back. Theory of Computing Systems, 2017, 61(1):191-232.

[4] Bertossi, L., Rizzolo, F. and Lei, J. Data Quality is Context Dependent. Proc. Enabling Real-Time Business Intelligence (BIRTE 2010). Springer LNBIP 84, 2011, pp. 52-67.

[5] Brewka, G., Eiter, T. and Truszczynski, M. Answer Set Programming at a Glance. Comm. of the ACM, 2011, 54(12):93-103.

[6] Chomicki, J. and Marcinkowski, J. Minimal-Change Integrity Maintenance Using Tuple Deletions. Inf. Comput., 2005, 197(1-2):90-121.

[7] Caniupan-Marileo, M. and Bertossi, L. The Consistency Extractor System: Answer Set Programs for Consistent Query Answering in Databases. Data \& Knowledge Engineering, 2010, 69(6):545-572.

[8] Eiter, T. and Mannila, H. Distance Measures for Point Sets and their Computation. Acta Informatica, 1997, 34:109-133.

[9] Grant, J. and Martinez, M.V. (eds.) Measuring Inconsistency in Information. College Publications, 2018.

[10] Grant, J. and Hunter, A. Analysing Inconsistent Information Using Distance-Based Measures. Int. J. Approx. Reasoning, 2017, 89:3-26.

\footnotetext{
${ }^{6}$ Certain (or skeptical) reasoning with repair programs for DCs with weak constraints is $\Delta_{2}^{P}(\log (n))$-complete in data complexity, i.e. in the size of the database [7] 12].
} 
[11] Kivinen, J. and Mannila, H. Approximate Inference of Functional Dependencies from Relations. Theoretical Computer Science, 1995, 149:129-149.

[12] Leone, N., Pfeifer, G., Faber,W., Eiter, T., Gottlob, G., Perri, S. and Scarcello, F. The DLV System for Knowledge Representation and Reasoning. ACM Trans. Comput. Logic., 2006, 7(3):499-562.

[13] Meliou, A., Gatterbauer, W., Moore, K. F. and Suciu, D. The Complexity of Causality and Responsibility for Query Answers and Non-Answers. Proc. VLDB, 2010, pp. 34-41.

[14] Rajamaran, A. and Ullman, J. Mining of Masssive Datasets. Cambridge Univ. Press, 2012.

[15] Staworko, S., Chomicki, J. and Marcinkowski, J. Prioritized Repairing and Consistent Query Answering in Relational Databases. Ann. Math. Artif. Intell., 2012, 64(2-3):209-246.

[16] Thimm, M. On the Compliance of Rationality Postulates for Inconsistency Measures: A More or Less Complete Picture. Künstliche Intelligenz, 2017, 31(1):31-39. 